\title{
EL CAPITAL SOCIAL DESDE UNA
}

\author{
APROXIMACIÓN ÉTICA
}

\section{SOCIAL CAPITAL FROM AN ETHICAL APPROACH}

MAURICIO BURBANO ALARCÓN ${ }^{1}$

Recibido: 1de noviembre de 2016

Aprobado: 20 de febrero de 2017 



\title{
EL CAPITAL SOCIAL DESDE UNA APROXIMACIÓN ÉTICA
}

\section{SOCIAL CAPITAL FROM AN ETHICAL APPROACH}

\author{
Mauricio Burbano Alarcón
}

Palabras clave: capital social, desarrollo, ética, epistemología.

Keywords: social capital, development, ethics, epistemology.

\section{RESUMEN}

El concepto de capital social ha generado interés en variadas disciplinas: ciencia política, economía, sociología, educación, sicología, etc.; sin embargo, poco se ha escrito sobre sus aspectos éticos. El presente artículo aborda dichos aspectos a partir de una doble aproximación. En primer lugar, se considerará el capital social desde las diversas perspec- tivas propuestas por sus principales teóricos (P. Bourdieu, J. Coleman y R. Putnam) a partir de una clarificación conceptual con énfasis en sus aspectos éticos particulares. En segundo lugar, se plantearán diversas consideraciones éticas sobre el capital social como concepto relacional concerniente con la confianza.

\section{ABSTRACT}

The concept of social capital has generated interest in varied disciplines: political science, economics, sociology, education, psychology, etc. However, li- ttle has been written about the ethical issues involved. This article addresses these issues from a dual approach. First, social capital is considered from various 
perspectives as proposed by its leading theorists (P. Bourdieu, J. Coleman and R. Putnam) from a conceptual clarification with emphasis on its particular ethical aspects. Secondly, various ethical considerations concerning social capital as relational concept with regard to trust will be taken into consideration.

\section{INTRODUCCIÓN}

El capital social se ha constituido en una categoría de análisis que ha incursionado en diversas disciplinas como la economía, sociología, ciencia política, educación, sicología, etc. El hecho de que esta categoría se ha empleado en diversos ámbitos implica que se le ha considerado como un instrumento de análisis válido. Con todo, este concepto tiene unas posibilidades y límites como cualquier otra categoría analítica de las ciencias sociales que separa, distingue y divide un fenómeno social para volverlo inteligible. Al respecto, ya existe una abundante literatura crítica sobre sus límites, por lo cual no se insistirá en ese aspecto. Más bien se abordará el capital social como un concepto que se define a partir de las relaciones de cooperación en distintos niveles (como pueden ser el familiar, vecinal, ciudadano, etc.) lo cual a su vez implica una acción o praxis que como tal puede ser abordada valorativa- mente desde un aspecto ético. De ahí la pertinencia de este artículo que pretende ser una aproximación al abordaje del capital social desde la ética.

El concepto de "capital social", si bien parte de la noción de relación, presenta matices de acuerdo a las distintas perspectivas teóricas consideradas, en donde cabe resaltar los aportes de Pierre Bourdieu, James Coleman y Robert Putnam. Quienes por primera vez abordaron de manera sistemática el capital social fueron P. Bourdieu y J. Coleman, por lo que pueden ser considerados los teóricos fundadores del capital social; mientras en el caso de R. Putnam, desarrolló su concepto a partir de Coleman (Häuberer, 2011: 35, 53). En este sentido, en un primer momento se consideran estas tres perspectivas teóricas, para luego -en un segundo momento- abordar de manera global el capital social desde una perspectiva ética. 


\section{P. BOURDIEU: CAPITAL SOCIAL COMO MANTENIMIENTO DE ESTATUS SOCIAL}

El capital social, según Pierre Bourdieu, es un "conjunto de recursos reales o potenciales que están ligados a la posesión de una red duradera de relaciones más o menos institucionalizadas de mutuo conocimiento y de inter-reconocimiento; en otras palabras, el pertenecer a un grupo como un conjunto de agentes que no solo están equipados con propiedades comunes (percibidas por el observador, por otros o por sí mismos) sino también están unidos por lazos permanentes y útiles" 1 (Bourdieu, 1980: 2). El capital social tiene que ver con los "recursos basados en la pertenencia a un grupo" (Bourdieu, 2000 [1986]: 148) que puede traer beneficios materiales y simbólicos propios de la pertenencia a un grupo. La red de relaciones no se daría de forma "natural" sino más bien se debe a "estrategias individuales o colectivas de inversión, consciente o inconscientemente dirigidas a establecer y mantener relaciones sociales que prometan, más tarde o más temprano, un provecho inmediato" (Bourdieu, 2000 [1986]: 151).

El capital social sería una forma de capital cercana a otras formas: capital simbólico y cultural. Estas formas de ca- pital social habrían sido frecuentemente ignoradas por cuanto son menos tangibles y por tanto más difíciles de medir que el capital económico. Ellas jugarían un rol importante en la transmisión de ventajas sociales a lo largo de las generaciones, por lo que al comprenderlas se podría sacar a la luz las relaciones de poder y, por tanto, mostrar las desigualdades producidas (Manza y Sauder, 2006). Este capital social se vería conservado y mantenido gracias a la homogeneidad del grupo, por lo que incorporar a un nuevo individuo a la red implicaría pasar por el escrutinio de la legitimidad. Así, actividades sociales como reuniones, recepciones, prácticas deportivas, lugares (escuelas, clubes, etc.) fomentarían el mantenimiento y acumulación de capital social manteniendo los límites del grupo. En otras palabras, el capital social no sería tan "social" en el sentido de que no está disponible como bien público, sino que sería usufructuable solamente para quien pertenece a un grupo específico con su respectivo un contexto específico de socialización que le proporciona un "hábitus" particular. De esta manera, el concepto de capital social en Bourdieu está asociado al mantenimien-

\footnotetext{
${ }^{1}$ Traducción propia.
} 
to de estatus social que a la larga conllevaría a mecanismos de mantenimiento y reproducción de la desigualdad social.

El monto de capital social que puede ser acumulado por un individuo depende del tamaño de la red de conexiones que puede ser movilizada y el volumen de capital que tienen los otros miembros de la red, ya se trate de capital simbólico, cultural o económico (Manza y Sauder, 2006: 558). En Bourdieu el capital social no se concibe de manera independiente, sino dentro de un juego de relaciones en el que los diversos tipos de capital son más o menos eficientes en un "campo" determinado desde el cual se ejercen un juego de fuerzas.
En cuanto a las críticas, al modelo de capital social de Bourdieu, si bien por un lado su noción de capital social intenta inscribirse dentro de una visión compleja -no simplista- de análisis de un hecho social, estaría anclado a un modelo estático de jerarquía social basado en las particularidades de la burguesía francesa de los años 60 y principios de los 70 (Field, 2008: 20, 22). Esto haría que Bourdieu considere el capital social únicamente en función de sus efectos de "exclusión" en donde las élites mantienen y reproducen sus privilegios. Es decir, solamente se consideraría el lado negativo del capital social como potencial de relaciones excluyentes.

\section{J. COLEMAN: CAPITAL SOCIAL COMO BIEN COMÚN}

James Coleman aborda inicialmente el capital social en el año 1988 al investigar los entornos educativos caracterizándolas como "redes cerradas". Esta característica posibilitaría que se generen un conjunto de expectativas y obligaciones que van más allá de la familia nuclear entre padres e hijos. Así, cada adulto puede usar su capital social de tal manera que otros adultos pueden ayudar a supervisar a sus hijos. Una red de relaciones cerrada tendría la utilidad de compensar desequilibrios en el poder de los actores (niños vs. padres; estudiantes vs. profesores). La parte más débil de la relación (niños y estudiantes) podría fortalecer así su posición en relación con el tipo más poderoso del actor gracias a que la red es cerrada (Coleman, 1990: 318). De allí se comprende que un niño en la escuela pueda recibir apoyo y protección de un adulto que no es su padre o madre.

En un segundo momento, Coleman incluye el "capital social" como una parte de su obra culmen: Foundations of Social Theory (1990) en la que desarrolla una teoría social tomando como presu- 
puesto la acción racional individual, estableciendo una teoría de los sistemas sociales en donde hay una interdependencia entre lo micro (individual) y lo macro (colectivo). Desde esta perspectiva, intenta dar cuenta de un proceso sociohistórico en el que han aparecido nuevos actores sociales. El medio natural de relaciones se daría como un "capital social primordial" asociado con aquellas agrupaciones que tradicionalmente han proporcionado capital social: la familia, el clan o el grupo de pertenencia. En este "contexto primordial" se establecerían los procesos de socialización con sus normas, regulación y protección de los distintos espacios de intercambio ya se trate del ocio, trabajo, religión, etc. Debido a los procesos de modernización del Estado y de la vida social, varias de las funciones que eran sustentadas por estructuras primordiales (con lazos inmediatos y cercanos) serían parcial o totalmente reemplazadas por una estructura intencional. Así, entidades estatales y corporaciones privadas van adquiriendo una mayor relevancia. El Estado asumiría el control y protección de la salud, la regulación del empleo y protección en caso de desempleo. Espacios antes exclusivos de la competencia familiar se van ampliando hacia la normalización externa como el caso de la educación y crianza de los niños. La "estructura primordial" se va desmoronando en sus funciones y van surgiendo "agentes corporativos" en los que no interviene solo el Estado, sino instituciones privadas con fines comerciales. Así, nos encontraríamos con una extensa oferta que se enfocaría estratégicamente (target) en atender distintas necesidades de la población de acuerdo a rangos de edad o de intereses, reemplazando así los lazos familiares y vecinales que antes posibilitaban las relaciones intergeneracionales (Coleman, 1990: 585). Si el medio social de los lazos primordiales referidos a la familia, al grupo étnico, clan y comunidad van siendo sustituidos por nuevos actores corporativos intencionalmente construidos, esta sustitución implicaría un "cambio cualitativo" en la forma de organización social (Coleman, 1990: 552, 651). Comprender las relaciones entre estos distintos actores sociales en los Estados modernos a partir del capital social desde esta perspectiva sistémica, permitiría relacionar lo micro y lo macro en la estructura social, posibilitando una mejor comprensión de las necesidades sociales de cara a la elaboración de políticas públicas.

El capital social, según Coleman, se define por su función: "No es una sola entidad, sino una variedad de entidades diferentes que tienen dos características en común: todas consisten en algún aspecto de la estructura social, y facilitan ciertas acciones de los individuos que están dentro de la estructura. Como 
otras formas de capital, el capital social es productivo, haciendo posible el logro de ciertos fines que no serían alcanzables en su ausencia" (Coleman, 1990: 301)². El capital social se distingue del capital físico y humano. El capital físico es tangible y se muestra en una forma material observable, el capital humano es menos tangible que el físico puesto que hace referencia a los conocimientos y habilidades adquiridas por un individuo. El capital social es el menos tangible de todos, porque está "encarnado" (embodied) en las relaciones sociales que se dan entre las personas (Coleman, 1990: 304). Todos estos capitales facilitan la actividad productiva, pero la característica del capital social es que se posibilita cuando las relaciones entre personas facilitan la acción puesto que, si hay relaciones de confianza, credibilidad e información veraz, estas facilitarán la acción de los individuos en la consecución de sus intereses.

Coleman concibe el capital social en términos de "estructura social" dentro de las relaciones sociales de confianza. Según Fukuyama, la confianza se puede definir como "la expectativa que surge dentro de una comunidad con un comportamiento ordenado, honesto y cooperativo, basada en normas comúnmente compartidas, por parte de los miembros de esa comunidad" (Fukuyama, 1995: 26). A diferencia de Bourdieu, para quien el capital social es exclusivo (bien privado), el capital social según Coleman es un bien público, es decir no constituye una propiedad privada de las personas que se benefician de él, ya que es un atributo que se encuentra inserto (embedded) o incorporado en la estructura social (Coleman, 1990: 315). Considerado de esta manera, el capital social sería un recurso importante que podría afectar a la calidad de vida no solamente en los aspectos monetarios, sino en cuestiones como la salud en cuanto bienestar físico y mental.

La mayoría de las formas de capital social se crean o destruyen como un "subproducto" (byproduct) de otras actividades (Coleman, 1990: 317). Esto haría posible que muchos de los beneficios de las acciones que traen capital social sean experimentados por distintos actores con diferentes propósitos. Aquí radicaría la dimensión pública del capital social puesto que podrían beneficiarse de él incluso individuos que no han tenido un interés directo en fomentarlo. Esta propiedad de ser una estructura de dimensión pública hace que el capital social también pueda "transformarse". Por ejemplo, una organización con un propósito definido puede constituirse en otras relaciones entre individuos, teniendo así un capital social disponible que ayuda para nuevos propósitos. Esta for-

${ }^{2}$ Traducción propia. 
ma transformada de capital social puede verse disuelta en otros elementos ya sea información potencial, normas, expectativas, obligaciones y relaciones de autoridad (Coleman, 1990: 312).

La creación y/o destrucción del capital social depende de tres factores principales: 1) el grado de apertura de la red; 2) la estabilidad; 3) la ideología. Las normas (sanciones/recompensas) se posibilitan si la red no es demasiado abierta, ya que de esta manera la confianza puede alcanzar el nivel necesario. La estabilidad es necesaria ya que las interrupciones de las relaciones sociales son destructivas para el capital social. La ideología implica valoraciones que tienen influencia en la acción y pueden comprender aspectos variados: perspectivas religiosas, ideas sobre lo que es justo, normas o códigos éticos o de conducta asociados a gremios profesionales, etc. Cabe resaltar que los códigos éticos profesionales no cum- plen solamente una función normativa, sino de confianza. Así, es necesario un capital social en la relación paciente-médico ya que en principio se confía en que el profesional médico no hará daño deliberadamente a su paciente, ya que se espera que siga los principios éticos de su profesión impuestos por el juramento hipocrático (Fukuyama, 1995: 26). De la misma manera, el médico espera que su paciente sea veraz al detallar sus síntomas y posibles hábitos que perjudican su salud. Asimismo, gracias a los idearios religiosos se puede generar capital social, como cuando una religión considera un valor atender a las necesidades de los demás, como en el caso de la caridad (virtud teologal cristiana). En contraste, un ideario económico y político que fomente la autosuficiencia y el individualismo no posibilitará la generación de capital social (Coleman, 1990: 320-321).

\section{R. PUTNAM: CAPITAL SOCIAL Y "VIRTUD CÍVICA"}

El capital social según Robert Putnam (2000: 19) hace referencia a las conexiones entre los individuos proporcionadas por las redes sociales, normas de reciprocidad y confianza que facilitan la acción y cooperación mutua. El capital social estaría relacionado con la "virtud cívica" facilitada por una red de relacio- nes sociales recíprocas de confianza posibilitadas por los contextos de participación. Los ciudadanos que forman parte de una comunidad cívica obedecen la ley que se han impuesto a sí mismos y tratan equitativamente a los otros y esperan ese mismo trato de los demás (Putnam, 1993: 111). Por otro lado, hay 
que notar que la "virtud cívica" implica salir de una concepción individualista de virtud, por lo que los individuos virtuosos pero aislados no constituyen una sociedad rica en capital social.

Según Putnam, el "compromiso cívico" se evidencia en el grado de participación en asociaciones de carácter voluntario que tienen sus más diversos fines: culturales, religiosos, ecológicos, filantrópicos, educativos, etc. La participación en diversas iniciativas asociativas mejoraría las normas colectivas y la confianza, derivando en efectos positivos para el bienestar común y la vida democrática. En este sentido, el capital social como compromiso cívico es un bien público (al igual que Coleman) que sería necesario para el bienestar general como son otros bienes públicos: el agua, la educación, etc. (Manza y Sauder, 2006: 559).

Robert Putnam considera que hay dos tipos de capital social: unión (bonding social capital) y puente (bridging social capital). Más adelante, Michael Woolcock (2001) introdujo un tercer tipo: enlace (linking social capital). A continuación, se describen las características de cada uno de ellos.

\section{Capital social de unión}

Este capital social tiene que ver con las identidades exclusivas u homogéneas de los grupos. Se encuentran ejemplos de este capital social en una gran variedad de agrupaciones como pueden ser asociaciones de padres de familia, grupos de estudio bíblico, asociaciones de carácter étnico, asociaciones de migrantes, etc. Este capital social está relacionado con los aspectos identitarios de un determinado grupo o comunidad a partir de distintas dimensiones: familiares, étnicas, religiosas, culturales, lingüísticas, etc. Estas dimensiones forman parte de la propia socialización del individuo, por lo que se puede afirmar que sin la presencia del capital social de unión sería imposible desarrollar las habilidades sociales básicas que permiten desenvolverse en un determinado contexto sociocultural. Con todo, en el caso de las asociaciones, el hecho de que utilicen un discurso que justifica y explica sus acciones solo en función de su grupo particular, no implica necesariamente que derive en un "gueto" ya que puede haber asociaciones que teniendo un discurso autorreferencial (como un indicio de un fuerte capital social de unión) tengan a la vez un capital social puente que les permita integrarse dentro del tejido asociativo de su territorio y una participación activa en el espacio público (Bolíbar, 2013: 132).

El capital social de unión también posibilita estrechar los lazos de solidaridad y reciprocidad dentro de la red social a la que pertenecen. Por ejemplo, 
las redes sociales densas de carácter étnico proveen un soporte sicológico y social para los menos favorecidos de una comunidad (Putnam, 2000: 22) en un contexto que en un principio debido a su escasa familiaridad con el mismo puede resultar hostil. La escuela como red social cerrada puede brindar apoyo y protección a los niños a partir de las propias normas y colaboración dentro de la misma. En todo caso, el hecho de que un grupo tenga normas, si bien puede ser un principio generador de capital social de unión, también establece principios de inclusión/exclusión, pudiendo fomentar restricciones a la libertad, exclusión de otros y fortalecimiento de barreras clasistas (aspecto abordado por Bourdieu). De ahí, la importancia de no tener únicamente elementos de unión, sino también puente.

\section{Capital social puente}

El capital social puente (bridging) se desarrolla cuando existen lazos de confianza y reciprocidad entre grupos diferenciados, posibilitando el flujo de información y conexión con recursos externos. Este capital social se manifiesta entre grupos que son diversos, ya sea por cuestiones étnicas, sociales, culturales, etc. Como ejemplos que posibilitan el "puente" están los movimientos civiles, grupos ecuménicos, asociaciones de migrantes en las que los miembros no son solo de una nacionalidad, etc. También pueden ser grupos relativamente homogéneos internamente pero que a la vez comparten con otros grupos como en el caso de asociaciones de colombianos que comparten actividades con ecuatorianos.

Las relaciones posibilitadas en contexto diferenciado no necesariamente son "simétricas", por lo que pueden presentar jerarquías. Así mismo, no se comparte necesariamente todo el universo valorativo o normativo, por lo cual la confianza puede verse afectada o puede presentarse de forma diferenciada y localizada en acciones específicas. Con todo, la diversidad no sería un impedimento para acceder a una red de recursos que se encuentran fuera de la red de relaciones cercanas (unión). En el aspecto operativo, este capital social se desarrolla gracias a la comunicación, acceso a información, actividades formativas, lúdicas, festivas, etc. siempre y cuando se establezcan entre grupos distintos. La presencia del capital social puente mostraría a una comunidad que no es endogámica, sino que incorpora la diversidad y se enriquece con el contacto exterior.

La relación entre grupos diferentes trae efectos positivos a la sociedad en general; por ejemplo, el incorporar nuevas ideas para atender de forma innovadora viejos problemas o necesidades locales, el enriquecimiento cultural 
que implica tener pueblos o ciudades diversas. Con todo, la diversidad puede ser fuente de conflicto si antes no se han tratado o resuelto las desigualda-

des estructurales. Por eso, la UNESCO recomienda "invertir en diversidad cultural y diálogo intercultural" no desde un exótico "folclorismo", sino atendiendo a los Objetivos de Desarrollo del Milenio (ODM) a partir de acciones concretas que tienen que ver con la erradicación de la pobreza, acceso a la educación, equidad de género, desarrollo sustentable, etc. (Unesco, 2009). En este mismo sentido, los Objetivos del Desarrollo Sostenible se proponen para el 2030 "reducir la desigualdad en y entre los países" (Objetivo 10) en donde se constata que la desigualdad no solo afecta el ámbito económico sino al "sentimiento de realización y autoestima de las personas" (ONU, 2016).

En cuanto a las posibilidades de generar capital social puente en contraste con el de unión, se requiere menos esfuerzo construir vínculos de "unión" entre personas con una misma cultura, tradiciones y lengua, mientras que construir relaciones "puente" en contexto de diversidad se requiere una mayor inversión en tiempo. Por esta razón, es necesario contextualizar cuando los medios de comunicación y las administraciones públicas se alarman al mirar que extranjeros forman un grupo diferenciado, preocupación que se agudiza si dichas agrupaciones poseen algún elemento religioso diferente de la mayoría, como el caso de musulmanes en zonas que tradicionalmente han sido cristianos. Antes de prejuzgar es necesario considerar que solamente a partir de un primer capital social (unión) se pueden establecer posteriormente vínculos más heterogéneos. Es decir, para que haya presencia de relaciones puente es necesario que antes haya vínculos de unión. Entender este proceso, puede hacer que las administraciones locales no miren con sospecha la presencia y manifestación de los aspectos religiosos de los inmigrantes (Mata y Giró, 2013). El temor surge al creer que los grupos de migrantes constituyen "enclaves" cuyos lazos fuertes los aíslan de la sociedad. La situación es mucho más compleja: ¿Los migrantes quieren "voluntariamente" aislarse en enclaves étnicos o se ven "forzados" a ello?

En el momento inicial de asentamiento, las minorías étnicas normalmente se congregan para desarrollar su propia infraestructura de apoyo y para enfrentar su nueva situación por lo cual pueden crear sus propias formas de circulación de información, etc. A partir de esa base, con el tiempo, pueden hacer conexiones más amplias, y así conseguir moverse en otros ámbitos. Cuando estos puentes son bloqueados por la discriminación racial y la escasez de 
oportunidades (laborales, educativas, etc.) los miembros de las minorías étnicas no tienen otra opción que centrar sus actividades en el estrecho círculo de su comunidad donde encuentran apoyo económico, social, religioso, etc. Esto a su vez incrementa las sospechas del grupo mayoritario que observa el desarrollo y crecimiento del enclave, lo cual refuerza la segregación y "extranjerización" en el caso del inmigrante. La segregación étnica se convierte así en una profecía autocumplida que a su vez genera discursos racistas y de exclusión por parte de la sociedad mayoritaria (Castles, 2000: 199).

\section{Capital social de enlace}

El capital social de enlace (linking) enfatiza las relaciones de personas, comunidades $\mathrm{o}$ asociaciones en un contexto participativo en donde el Estado tiene su presencia a partir de una variada institucionalidad a nivel local y nacional. Este capital social posibilita que la ciudadanía y sus organizaciones sociales puedan manifestar sus planteamientos y propuestas de cara a la institucionalidad estatal. La presencia o ausencia de capital social de enlace puede evidenciar el grado de interés oficial institucional estatal de acercarse a ciertos grupos de personas en situación de vulnerabilidad (como pueden ser las asociaciones de refugiados y migrantes irregulares). Por otro lado, no es requisito indispensa- ble que una organización social sea de carácter formal para acceder a la institucionalidad. Este puede ser el caso de asociaciones que, a pesar de ser informales, mantienen un liderazgo que cuenta con una dinámica red de contactos, que logra abrir puertas institucionales de carácter local (como municipios). En otras palabras, no hay que asumir a priori que una agrupación de carácter informal no podrá tener un capital social de enlace.

Los tres tipos de capital social vistos anteriormente pueden tener diferentes combinaciones que producirán diversos resultados (Field, 2008: 46). En este sentido, no basta considerar solamente el capital social de unión y puente, sino es necesario incorporar el capital social de enlace que puede ser determinante para que dos grupos con parecido capital social de unión y puente presenten resultados finales distintos. Como insiste Woolcock (2001: 13) no hay que perder de vista el contexto institucional en el que se enmarcan las redes sociales. La presencia o escasez de capital social no se entienden sin tener en cuenta el entorno más amplio en el que las comunidades pueden lograr unas relaciones complementarias con la institucionalidad del Estado o, por el contrario, pueden ser totalmente ignoradas.

Las tres perspectivas de "capital social" tienen en común que constituyen un concepto "relacional" (Narayan, 
1999: 6). Se puede identificar una tipología general en la que se distinguen tres componentes básicos del capital social: a) una red; b) conjunto de normas; c) sanciones. Es decir, el capital social consiste en una red de personas que comparten unas normas, valores, expectativas que a su vez conllevan sanciones (premios o castigos) que ayudan a mantener dicha red y sus normas (Halpern, 2005: 10, 39). Las normas y sanciones no necesariamente tienen que estar escritas. Por ejemplo, en el caso del capital social de un barrio, dichas normas pueden presentarse implícitamente sin que haya algún registro o acuerdo escrito: no hacer ruido en la noche, ser cortés con el vecino, limpiar la vereda que corresponde a nuestra propiedad. Las sanciones igualmente pueden presentarse de modo formal (al quebrantar un reglamento o ley explícitamente estipulado) o informal. El que haya sanciones informales no implica que sean menos efectivas (Halpern, 2005: 11). En el caso de un barrio, la sanción frente a una norma incumplida puede ir desde llamar la atención directamente, intercambiar palabras airadas o emprender una acción concreta como poner una denuncia en el municipio. Sin embargo, es común que las sanciones sean indirectas y sutiles como por ejemplo, a través del chis- me crear una mala reputación de cierto vecino (Halpern, 2005: 11). La otra cara de la sanción es el "premio" recibido por el cumplimiento de la norma. Puede presentarse como una felicitación, aumento de reputación, etc. Estos tres componentes del capital social podrían ser usados para analizar todo tipo de comunidad, tomando en cuenta que las redes, normas y sanciones pueden tener aspectos formales (contemplados en estatutos) y aspectos informales que son implícitos y tácitos (Halpern, 2005: 12). El hecho de que un grupo o una comunidad tenga y acepte unas normas comunes genera un sustrato para el mantenimiento de la confianza. Por otro lado, no hay que olvidar que hay factores sociales que pueden condicionar la generación de capital social, especialmente en comunidades que han sido excluidas a lo largo de la historia. En este sentido, no se puede afirmar fácilmente que una comunidad debido a su "falta de emprendimiento" no accede a los recursos necesarios provenientes del capital social. Hay elementos contextuales históricos condicionantes (como las inequidades de género, el racismo y la xenofobia) que dificultan el acceso a recursos, lo que provocará que se perpetúen las inequidades sociales. 


\section{CONSIDERACIONES ÉTICAS SOBRE EL CAPITAL SOCIAL}

En los apartados anteriores hemos visto cómo las distintas perspectivas de capital social de Pierre Bourdieu, James Coleman y Robert Putnam conllevan diversos aspectos valorativos lo cual implica entrar en consideraciones éticas. Siguiendo a Patricia Illingworth, se puede afirmar que el capital social cumple con los criterios de un concepto moral (Illingworth, 2012: 390). Cuando Adela Cortina se pregunta: ¿Para qué sirve la ética? responde a partir de criterios que se relacionan con el capital social. Cortina afirma que la ética sirve "para abaratar costes en dinero y sufrimiento en todo aquello que depende de nosotros, e invertirlo en lo que vale la pena, sabiendo priorizar" (Cortina, 2013: 26), y precisamente el capital social a partir de las normas y redes de confianza implica un abaratar costes de dinero y de sufrimiento y su presencia puede ser determinante para fomentar el bienestar general de una comunidad en donde se incluyen aspectos relacionados con la salud pública, sentido comunitario de pertenencia, solidaridad, etc. En definitiva, "si fuera posible un mundo en que contara como moneda corriente la confianza en las familias, las escuelas, las organizaciones y las instituciones, la vida sería infinitamente más barata. Y no solo en dinero [...] sino también y sobre todo en muer- tes prematuras, en vidas destrozadas, en conflictos, en eternos procesos judiciales de final incierto, en venganzas, rencores, en papeleos odiosos y en ese coste que varía más o menos, pero que suelen acabar pagando los peor situados (Cortina, 2013: 14). Así, la confianza, además de ser una fuente de generación de capital social, es importante ya que se constituye como el "principal 'recurso moral' de una sociedad" (Cortina, 2013: 23).

El capital social se presenta como subproducto de otras actividades, pero no cualquier tipo de actividades, sino aquellas que implican "relacionalidad". Es decir, las exigencias del capital social implicarían el esfuerzo de ir más allá del interés propio. El capital social se posibilita comunitariamente ya que no puede ser producido por un individuo aislado. El congregarse en diversas organizaciones sociales (como puede ser una asociación de migrantes, trabajadores, padres de familia) implica un beneficio que puede extenderse a los miembros más frágiles de sus respectivas agrupaciones como pueden ser los migrantes irregulares, obreros con salarios bajos o los niños con mayor necesidad de atención escolar. En este sentido, Illingworth considera que es una obligación moral promover la construcción de capital social para llegar a los diversos miembros de la comu- 
nidad que de otra manera podrían ser excluidos, y cada uno de nosotros estaría obligado moralmente a ampliar nuestro círculo de amigos, asociados y colegas para incluir a personas de diferentes culturas y grupos socioeconómicos (Illingworth, 2012: 395). Por otro lado, si la generación de capital social implica salir del ámbito individual para salir al ámbito público, resulta pertinente la noción de "virtudes públicas" propuesta por Victoria Camps (1990) para quien -siguiendo a los filósofos clásicos griegos- la ética implica una formación del carácter que a su vez se relaciona con el ámbito público al tener en cuenta valores como la responsabilidad, tolerancia y solidaridad. Estas virtudes públicas son relevantes para la vida democrática, para la generación de confianza, y posibilitan la generación de capital social.

El capital social puede tener una dimensión transnacional que supere las barreras del estado-nación. Se puede generar capital social en comunidades migrantes transnacionales que gracias a sus lazos de reciprocidad, confianza y obligaciones compartidas pueden brindar un aporte al desarrollo de sus comunidades de origen. Otro caso de capital social, que va más allá de un estado-nación, son las experiencias de voluntariado internacional que posibilitan contacto con otros contextos alejados del terruño propio que permiten valorar otras concep- ciones de vida y sensibilizarse respecto a problemáticas de otras latitudes. Además, las actividades propias de un voluntariado (ya sea internacional o local) pueden crear un "círculo virtuoso"' de interacción social en la que los participantes se involucran con la población local, crean confianza y construyen un sentido de ciudadanía compartida (Illingworth, 2012: 400). Esta interrelación podría tener consecuencias políticas en búsqueda de eliminar prejuicios y fomentar la paz y el desarrollo. En este sentido, las relaciones y confianza en un contexto de diversidad deberían conllevar una visión particular de corresponsabilidad que a su vez implique una visión de justicia en el sentido de que es necesario que haya una distribución de cargas y beneficios en la sociedad en donde los miembros más débiles y vulnerables no sean abandonados a su suerte.

El capital social también puede verse influenciado por los aspectos normativos de un Estado lo que a su vez se relaciona con aspectos valorativos de carácter ético. Este es el caso de iniciativas subvencionadas por gobiernos locales o nacionales que incentivan pasantías y voluntariado a nivel nacional o internacional. O subvenciones públicas para organizaciones sociales (asociaciones) que pueden ser canalizadas para fomentar las relaciones sociales en contexto de diversidad y procesos de inclusión social. 
Además, se pueden presentar aspectos normativos y legales que fomenten una ética del cuidado y la solidaridad, promoviendo así el capital social. Con todo, la otra cara de la moneda se la encuen- tra en normativas o leyes que privan de derechos básicos a grupos específicos y desestimulan la solidaridad ${ }^{3}$, fomentando de esta manera la exclusión con la consecuente pérdida de capital social.

\section{CONCLUSIONES}

El "capital social" presenta distintos matices dependiendo de la perspectiva teórica abordada, que a su vez nos presenta diversos aspectos valorativos. Pierre Bourdieu maneja un concepto de capital social que se basa en la reproducción de los privilegios. Desde esta perspectiva, el capital social se enmarcaría dentro de un modelo de jerarquías sociales estático (Field, 2008: 22) que haría que sea exclusivamente un "bien privado" del que pueden usufructuar únicamente las élites. En este sentido, desde una perspectiva valorativa, el capital social no sería "social" sino más bien se relacionaría con una reciprocidad basada en intereses egoístas. En contraste, James Coleman considera al capital social como un "bien público" y lo enmarca dentro de una "teoría social general" de carácter sistémico que quiere dar cuenta de los actores y procesos sociales contemporáneos. El capital social se encontraría presente en la estructura social y se relaciona con redes sociales basadas en la confianza. Siguiendo a Coleman, en Robert Putnam el capital social es un bien público, aunque su perspectiva teórica pone el énfasis en la "virtud cívica" que se vuelve operativa cuando se generan organizaciones sociales de carácter voluntario. Esta perspectiva asume de una u otra manera aspectos que se asocian generalmente a los valores democráticos: libertad de expresión, libertad de asociación, convivencia, etc.

Más allá de las distintas perspectivas del capital social se trata de un concepto relacional que como tal conlleva una perspectiva ética de acercamiento al "otro". Cabe preguntarnos cuáles son nuestras redes, pertenencias comunitarias o grupales. Y si estamos dispuestos a ampliar dichas redes en donde se incluya al otro diverso ya sea por su condición social, ideología política, condición migratoria, pertenencia religiosa, etc. Este acercamiento al otro diverso implica una

\footnotetext{
${ }^{3}$ Por ejemplo, el caso de la penalización de comportamientos relacionados con la hospitalidad a personas
} inmigrantes que se encuentran en situación irregular. Ver: http://www.salvemoslahospitalidad.org/ 
acción consciente y voluntaria que nos de ser un extraño, deja de ser un extranpermita salir de nuestras comodidades jero para convertirse en un compañero y lugares comunes. De esta manera, am- de camino, lo cual implica aproximarnos pliaremos nuestros círculos de confianza a una postura ética del cuidado y la soliya que el "otro" deja de ser un ajeno, deja daridad. 


\section{BIBLIOGRAFÎA}

Bolíbar, M. (2013). Redes asociativas, inmigración y participación. Una aproximación empírica a los vínculos entre capital social y participación asociativa. REDES, 24(1), 114-136.

Bourdieu, P. (1980). Le capital social: notes provisoires. Actes De La Recherche En Sciences Sociales, 31, 2-3.

Bourdieu, P. (2000 [1986]). Las formas de capital. En Bourdieu, P. (2000 [1986]). Poder, derecho y clases sociales (2da ed., pp. 131-164.). Bilbao: Desclée de Brouwer.

Castles, S. (2000). Ethnicity and Globalization: From Migrant Worker to Transnational Citizen. London: Sage.

Coleman, J. (1990). Foundations of Social Theory. Cambridge: Harvard University Press.

Camps, V. (1990). Virtudes públicas. Madrid: Espasa-Calpe.

Cortina, A. (2013). ¿Para qué sirve realmente la Ética? Barcelona: Paidós.

Field, J. (2008). Social Capital (2nd ed.). London: Routledge.

Fukuyama, F. (1995). Trust: Social Virtues and the Creation of Prosperity. New York: The Free Press.

Halpern, D. (2005). Social Capital. Cambridge MA: Polity Press.
Häuberer, J. (2011). Social Capital Theory. Towards a Methodological Foundation. Wiesbaden: VS Verlag für Sozialwissenschaften.

Illingworth, P. (2012). Ethics and social capital for global well-being. International Review of Economics, 59, 389-407.

Manza, J., \& Sauder, M. (2006). Social Capital. En B. Turner (Ed.), The Cambridge Dictionary of Sociology (pp. 557-559.). Cambridge: Cambridge University Press.

Mata, A. y Giró, J. (2013). Reflexiones sobre la influencia de los componentes religiosos en el asociacionismo inmigrante. Revista Internacional de Sociología (RIS), 71, No. extra 1 - junio, pp. 117-140.

Narayan, D. (1999). Bonds and Bridges: Social Capital and Poverty. Washington, DC: World Bank.

ONU (2016). Objetivos de Desarrollo Sostenible. Organización de las Naciones Unidas. Recuperado de http://www.un.org/sustainabledevelopment/es/

Putnam, R. D. (1993). Making Democracy Work. Princeton, NJ: Princeton University Press.

Putnam, R. D. (2000). Bowling Alone: The Collapse and Revival of American 
Community. New York: Simon \& Woolcock, M. (2001). The place of social Schuster.

capital in understanding social

UNESCO (2009). Investing in Cultural Diand economic outcomes. Isuversity and Intercultural Dialogue $m a, 2(1), 11-17$.

- World Report. Paris: United Nations Educational, Scientific and Cultural Organization. 